\title{
Performance Investigation of Spatial Modulation Systems Under Non-Stationary Wideband High-Speed Train Channel Models
}

\author{
Yu Fu, Cheng-Xiang Wang, Senior Member, IEEE, Ammar Ghazal, \\ el-Hadi M.Aggoune, Senior Member, IEEE, and Mohammed M. Alwakeel, Senior Member, IEEE
}

\begin{abstract}
In this paper, the bit error rate (BER) performance of a new multiple-input-multiple-output (MIMO) technique, named spatial modulation (SM), is studied under a novel nonstationary wideband high-speed train (HST) channel model in different scenarios. Time-varying parameters obtained from measurement results are used to configure the channel model to make all results more realistic. A novel statistic property called the stationary interval in terms of the space-time correlation function (STCF) is proposed to describe the channel model's time-varying behavior. The accurate theoretical BER expression of SM systems is derived under the time-varying wideband HST channel model with the non-ideal channel estimation assumption. Simulation results demonstrate that the BER performance of SM systems shows a time-varying behavior due to the nonstationary property of the employed HST channel model. The system performance can maintain a relative stationary status within the specified stationary interval. It can also be observed that the BER performance of SM systems under the HST channel model is mainly affected by the correlation between sub-channels, inter-symbol-interference, Doppler shift, and channel estimation errors.
\end{abstract}

Index Terms-Spatial modulation, high-speed train channels, non-stationary channel models, bit error rate, stationary interval, space-time correlation function.

\section{INTRODUCTION}

$\mathbf{I}$ $\mathrm{N}$ recent years, high-speed train (HST) systems with the operating speed of more than $250 \mathrm{~km} / \mathrm{h}$ have gained significant developments. It was reported that the maximum speed of HSTs can easily reach $500 \mathrm{~km} / \mathrm{h}$ within the next decade and the passenger number will drastically increase [1]. Consequently, there is a great demand for high data rate and reliable communication services [2], [3]. However, the existing wireless communication systems (including the forthgeneration $(4 \mathrm{G})$ system) can only support mobile speed of up

The authors gratefully acknowledge the support of this work from the EPSRC TOUCAN project (Grant No. EP/L020009/1), the EU H2020 5G Wireless project (Grant No. 641985), the EU FP7 QUICK project (Grant No. PIRSES-GA-2013-612652), the Ministry of Science and Technology of China through the 863 Project in 5G (Grant No. 2014AA01A706), and the SNCS Research Center, University of Tabuk, Saudi Arabia.

Y. Fu, C.-X. Wang (corresponding author), and A. Ghazal are with the Institute of Sensors, Signals and Systems, School of Engineering and Physical Sciences, Heriot-Watt University, Edinburgh, EH14 4AS, U.K. C.-X. Wang is also with School of Information Science and Engineering, Shandong University, Jinan, 250100, China, and an Affiliate Researcher of the SNCS Research Center, University of Tabuk, Saudi Arabia (e-mail: $\{y$.fu, chengxiang.wang, ag289\}@hw.ac.uk).

e. H. M. Aggoune and M. M. Alwakeel are with the SNCS research center, University of Tabuk, Tabuk, Saudi Arabia (e-mail: \{haggoune.sncs, alwakeel\}@ut.edu.sa). to $250 \mathrm{~km} / \mathrm{h}$. Thus, reliable wireless communication services for future HST systems have been considered as an important issue for the fifth-generation $(5 \mathrm{G})$ wireless communication system [4].

Compared with conventional communication systems, the HST wireless communication system suffers more challenges such as larger Doppler shift. Besides, the handover of HST users can happen more frequently, which will result in higher probability of handover failure and connection loss. Also, as cabins of HSTs are usually built with metal like aluminum, there will be huge penetration losses when signals get into carriages [5]. In order to solve these problems, many new cellular architectures such as the distributed antenna system (DAS) [6]-[8] and the mobile relay station (MRS) [9], [10] (or mobile Femtocell [4], [11]) have been investigated. In this paper, the MRS solution with multiple antenna elements are considered as it has been adopted by some widely used standardized wireless channel models such as the WINNER II [12] model and the International Mobile Telecommunications Advanced (IMT-A) model [13] for high mobility scenarios. We consider a downlink system, where signals from a base station (BS) will firstly be received by the MRSs distributed on the surface of HST carriages in order to avoid huge penetration losses. MRS signals will then be passed to access points within carriages through cables and radiate to on-board mobile stations (MSs). MSs will communicate with the MRS rather than with the BS directly, which can significantly improve the link quality due to short distances and the avoidance of penetrate loss.

Besides new cellular architectures, novel physical layer transmit technologies should also be used to improve the service quality of HST wireless communication systems. Among various technologies, a multiple-input-multiple-output (MIMO) technology named the spatial modulation (SM) [14] has attracted lots of research interest in recent years due to its outstanding performance in enhancing spectral efficiency and reducing system complexity [14]-[18]. The basic idea for SM is that antenna indices can be used to carry information, and at each time instant, only one antenna is activated. As only a single antenna is active at each time instant, the interchannel-interference (ICI) which is treated as a big problem for conventional MIMO technologies, can be completely avoided. SM can significantly reduce the system complexity [14] [18]. In recent years, there have been fruitful achievements in the research work of SM technologies. For example, the 
generalized SM was proposed in [19], [20], which employs a subgroup of antennas to transmit signals and can further improve the system efficiency. SM can also be combined with other MIMO technologies such as the space-time coding. Related research works were reported in [21]-[23]. Beside radio frequency wireless communications, SM has also been used in optical wireless communication systems [24], [25].

In order to design and test communication technologies for HST systems, channel models that can accurately capture the underlying propagation characteristics should be used. Research work for channel measurement and channel modeling on HST scenarios can be found in [12], [13], [26]-[30]. Most of these aforementioned channel models assumed to fulfil the wide-sense stationary (WSS) condition in order to reduce the complexity. However, this assumption is not sufficiently accurate in HST scenarios, as shown by measurement results in [31]. It is therefore necessary to model HST channels with the non-stationary assumption. The first non-stationary regular-shaped geometry-based stochastic model (RS-GBSM) for HST scenarios was proposed in [32], [33], considering the distance between the transmitter $(\mathrm{Tx})$ and the receiver $(\mathrm{Rx})$ as time-varying. In [34], [35], this model was further improved by involving more time-varying parameters such as angles of arrival (AoAs) and angles of departure (AoDs).

The performance of SM so far has only been investigated for some simple fix-to-mobile (F2M) channel models, such as Rayleigh, Rician, and Nakagami models [14]-[16], [36], and a vehicle-to-vehicle (V2V) channel model [37]. However, all of these channel models were WSS narrowband channel models. Therefore, the influence of inter-symbol-interference (ISI) and time-varying channel on the bit error rate (BER) performance of SM systems cannot be investigated. In order to fill this research gap, this paper will investigate the BER performance of SM systems using the novel non-stationary wideband HST MIMO channel model proposed in [34] with channel estimation errors. To be realistic to the real environment, this channel model is configured using time-varying parameters from measurement results reported in [28]. The impact of estimation delay and Doppler frequency on the BER performance are studied both by theoretical analysis and simulations.

In the following, we can highlight the main contributions and novelties of this paper as follows.

1) We comprehensively investigate the BER performance of SM systems under a novel HST non-stationary wideband channel model. The non-ideal channel estimation case is considered. An accurate theoretical BER expression is derived to compare with all simulation results. BER simulation results have been comprehensively analyzed together with the investigation of spacetime correlation function (STCF). The link between the BER performance and channel statistic properties is established.

2) We propose a new statistical property: stationary interval in terms of STCF, and investigate the BER performance SM systems in terms of this property. The aim to propose this statistical property is to accurately and straightforwardly describe the non-stationary behavior of the HST channel model, especially to find the time interval within which the channel can be treated as relatively stationary.

The remainder of this paper is organized as follows. In Section II, a review of SM systems is given. In Section III, the wideband HST channel model and related important statistical properties including a novel statistic property are described. The theoretical expression of the BER for SM under the nonstationary HST wideband channel model is derived in Section IV. In Section V, simulation results are presented and analyzed. Finally, conclusions are drawn in Section VI.

\section{Spatial Modulation Systems}

The detailed idea of SM systems has been introduced in [14]. Let us assume there is a MIMO system using $N_{t}$ transmit antennas at the Tx and $N_{r}$ receive antennas at the Rx. The $M$ order quadrature-amplitude modulation (QAM)/phaseshift keying (PSK) modulation scheme is used as the digital modulator. At each time instant, $m=\log _{2}\left(N_{t}\right)+\log _{2}(M)$ bits can be transmitted, where $m$ is defined as the spectral efficiency of the SM system in bits/symbol. In this paper, we present the block diagram of the SM system in Fig. 1, where a system using 4 transmit antennas and the QPSK modulation scheme is considered. Let us assume the bit sequence 0001 is transmitted, the first two bits 00 are used to select Tx 1 as the transmit antenna and carried by the antenna index. The other two bits 01 are mapped to the complex symbol $S_{2}$ based on the constellation diagram. Then, the symbol is radiated by Tx1 through the channel $\boldsymbol{H}$. At the Rx side, the index of the active transmit antenna and the transmitted symbol $S_{2}$ are jointly estimated to retrieve the original message [15].

Mathematically, this procedure can be expressed as follows. Let us use $S$ to denote the transmit vector at the Tx side, which is a $N_{t} \times 1$ vector. At each time instant, there is only one Tx antenna active, thus, only one element in $\boldsymbol{S}$ is non-zero. $\boldsymbol{S}$ can be further expressed as $\boldsymbol{S}=[0,0, . ., S, . .0,0]_{N_{t}}^{T}$, where $[.]^{T}$ denotes transpose operation.

At Rx, the received $N_{r} \times 1$ vector $\boldsymbol{y}(t)$ can be written as:

$$
\boldsymbol{y}(t)=\boldsymbol{H}(t, \tau) \otimes \boldsymbol{S}(t)+\boldsymbol{n}(t),
$$

where $\otimes$ denotes the convolution operation, $\boldsymbol{n}(t)$ is the $N_{r} \times 1$ complex additive white Gaussian noise (AWGN) vector, with real and imaginary parts both having a double-sided power spectral density equal to $N_{0} / 2$ [14]. In addition, $\boldsymbol{H}(t, \tau)$ is the $N_{r} \times N_{t}$ channel matrix, which can be further defined as

$$
\boldsymbol{H}(t, \tau)=\left[\begin{array}{ccc}
h_{1,1}(t, \tau) & \ldots & h_{1, N_{t}}(t, \tau) \\
h_{2,1}(t, \tau) & \ldots & h_{2, N_{t}}(t, \tau) \\
\ldots & h_{q, p}(t, \tau) & \ldots \\
h_{N_{r}, 1}(t, \tau) & \ldots & h_{N_{r}, N_{t}}(t, \tau)
\end{array}\right],
$$

In (2), $h_{q, p}(t, \tau)$ is the channel impulse response between the $p$ th Tx and the $q$ th Rx. The detailed procedure to generate channel impulse responses will be introduced in Section III.

In our system, we assume that the Tx and Rx have perfect time and frequency synchronization. The Rx will jointly detect 


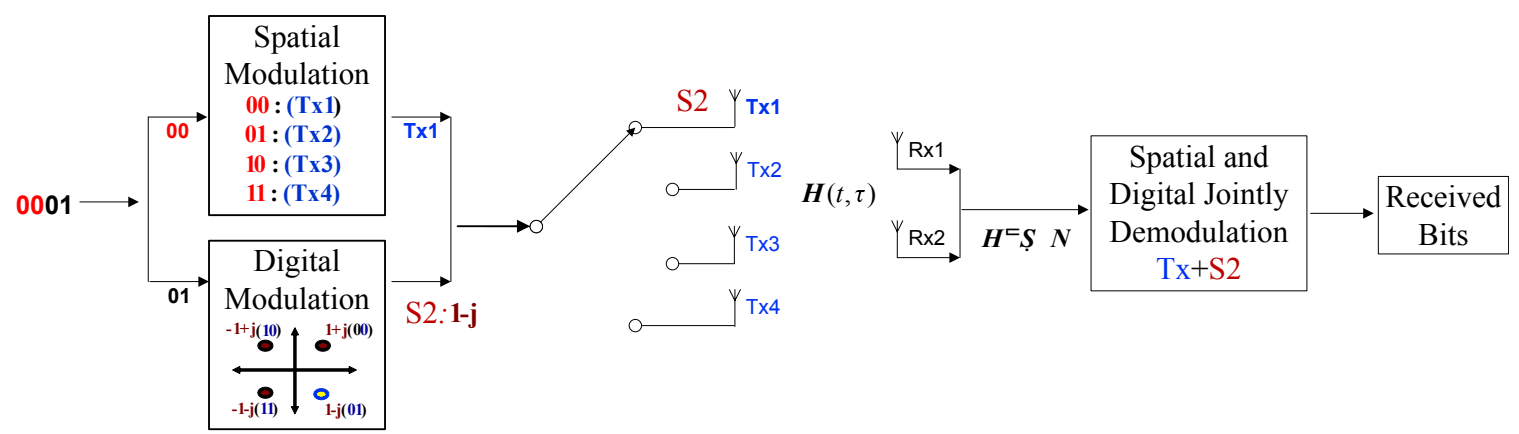

Fig. 1. Block diagram of SM system under mobile fading channels.

the index of the active transmit antenna and the modulation symbol with the estimated channel matrix $H_{\text {est }}$. The optimum maximum likelihood (ML) detector proposed in [15] is employed to estimate the transmit antenna index $\hat{p}$ and the transmitted data symbol $\hat{s}$ as

$$
\begin{gathered}
{[\hat{p}, \hat{k}]=\arg \min _{p, k}\left(\left\|y-H_{\text {est }} \otimes S_{k}\right\|_{F}^{2}\right),} \\
p \in\left\{1: N_{T}\right\} \text { and } k \in\{1: M\},
\end{gathered}
$$

where $\|\cdot\|_{F}$ denotes the Frobenius norm.

\section{THE NON-STATIONARY WIDEBAND HST CHANNEL MODEL}

In this paper, the HST system is assumed to use MRS to improve the wireless service quality. Thus, the wireless communication link between MSs and the BS is divided into two parts: BS to MRS and MRS to MS. Correspondingly, there are two types of wireless channels existing: the outdoor channel between BS and MRS, the indoor channel between MRS and MS, which are illustrated in Fig. 2. The indoor channel is similar to a conventional indoor scenario, which has already been widely investigated. In this paper, only the outdoor channel will be further studied.

Let us assume a MIMO HST system equips $N_{t}$ transmit and $N_{r}$ receive antenna elements. The BS is located on the track-side where a minimum distance between the BS and the track denoted as $D_{\min }=50 \mathrm{~m}$ [12] is considered. The time-varying distance between the BS and MRS is defined as $D_{s}(t)=\sqrt{\left(D_{\min }^{2}+D^{2}(t)\right)}$, where $D(t)$ stands for the projection of $D_{s}(t)$ on the railway track plane. Fig. 3 illustrates the abstracted multiple-confocal-ellipses GBSM. These ellipses represents multiple taps of the channel model. For different taps, corresponding propagation cases including direct links represented by line-of-sight (LoS) components, and single reflected signals represented as single-bounced (SB) rays, are considered. In this paper, a $2 \times 2$ MIMO channel model is used as an example as shown in Fig. 3, whose necessary parameters are illustrated in Table I.

Let us assume there are $N_{i}$ effective scatterers on the $i$ th ellipse (meaning the $i$ th tap), where $i=1,2, \ldots, I$, and $I$ is the total number or taps. Let $a_{i}(t)$ and $s^{\left(n_{i}\right)}$ denote the semi-major axis of the $i$ th ellipse and the $n_{i}$ th $\left(n_{i}=1, \ldots, N_{i}\right)$ effective scatterer respectively. Based on the geometric relationship, the

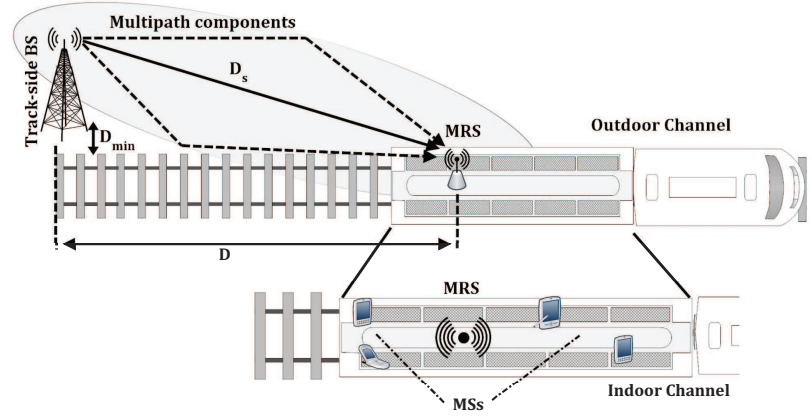

Fig. 2. A HST communication system deploying MRSs [34].

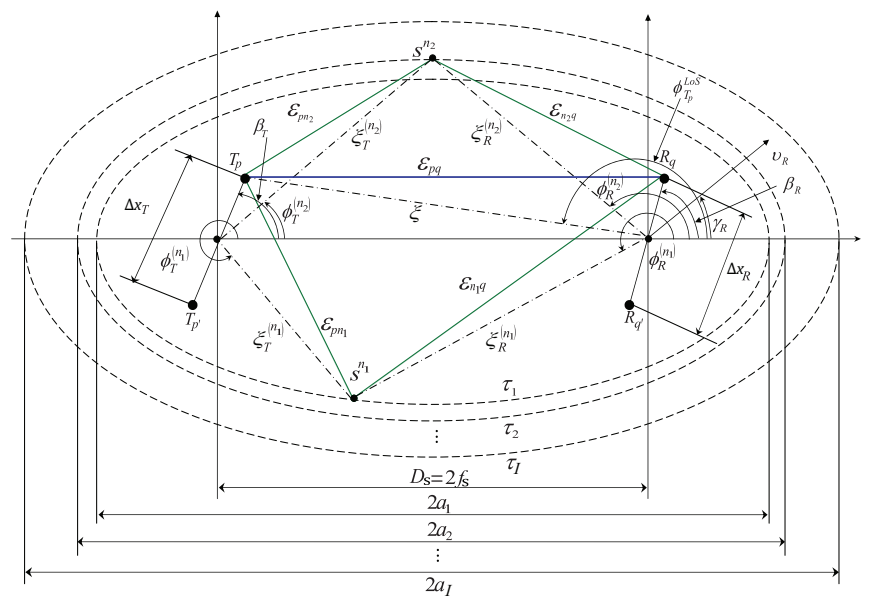

Fig. 3. The GBSM for a wideband MIMO HST channel [34].

time-varying semi-minor axis of the $i$ th ellipse can be expressed as $b_{i}(t)=\sqrt{a_{i}^{2}(t)-f_{s}^{2}(t)}$, where $f_{s}(t)=D_{s}(t) / 2$ denotes the half distance between the two foci of ellipses. The tilt angles of the BS and MRS antenna arrays are represented by $\beta_{T}$ and $\beta_{R}$, respectively. The MRS is assumed to move with the speed of $v_{R}$ in the direction determined by the angle of motion $\gamma_{R}$. The AoA of the wave traveling from an effective scatterer $s^{\left(n_{i}\right)}$ to the MRS is denoted by $\phi_{R}^{\left(n_{i}\right)}(t)$. The AoD of the wave that reflected through the effective scatterer $s^{\left(n_{i}\right)}$ is denoted by $\phi_{T}^{\left(n_{i}\right)}(t)$, while $\phi_{T_{p}}^{\mathrm{LoS}}(t)$ is the AoA of a $\operatorname{LoS}$ path.

The complex space-time-variant channel impulse response 
TABLE I

DEFINITION OF PARAMETERS IN FIG. 3 [34].

\begin{tabular}{|c|l|}
\hline Parameters & Definition \\
\hline$D_{s}(t)$ & distance between the BS and MRS \\
\hline$f_{s}(t)$ & half length of the distance between the two foci of ellipses \\
\hline$a_{i}(t), b_{i}(t)$ & semi-major axis and semi-minor axis of the $i$ th ellipse, respectively \\
\hline$v_{R}, \gamma_{R}$ & MRS speed and angle of motion, respectively \\
\hline$\Delta x_{T}, \Delta x_{R}$ & antenna element spacings of the BS and MRS \\
\hline$\beta_{T}, \beta_{R}$ & tilt angles of the BS and MRS antenna arrays in the x-y plane (relative to the x-axis), respectively \\
\hline$\phi_{T_{p}}^{\text {LoS }}(t), \phi_{R}^{\left(n_{i}\right)}(t)$ & AoA of the LoS path and AoA of the wave travelling from an effective scatterer $s^{\left(n_{i}\right)}$ to the MRS \\
\hline$\phi_{T}^{\left(n_{i}\right)}(t)$ & AoD of the wave that impinges on the effective scatterer $s^{\left(n_{i}\right)}$ \\
\hline$\xi, \xi_{T}^{\left(n_{i}\right)}(t)$, and $\xi_{R}^{\left(n_{i}\right)}(t)$ & distances $d\left(T_{p}\right.$, MRS $), d\left(\mathrm{BS}, s^{\left(n_{i}\right)}\right)$, and $d\left(s^{\left(n_{i}\right)}\right.$, MRS $)$, respectively \\
\hline$\varepsilon_{p q}, \varepsilon_{p n_{i}}, \varepsilon_{n_{i} q}$ & distances $d\left(T_{p}, R_{q}\right), d\left(T_{p}, s^{\left(n_{i}\right)}\right)$, and $d\left(s^{\left(n_{i}\right)}, R_{q}\right)$, respectively \\
\hline
\end{tabular}

represents the link between the $p$ th $\left(p=1, \ldots, N_{t}\right)$ element of the Tx, $T_{p}$, and the $q$ th $\left(q=1, \ldots, N_{r}\right)$ element of the Rx, $R_{q}$, can be expressed as $h_{p q}(t, \tau)=\sum_{i=1}^{I} h_{i, p q}(t) \delta\left(\tau-\tau_{i}\right)$, where $h_{i, p q}(t)$ and $\tau_{i}$ are the complex space-time-variant coefficients and the propagation delay of the $i$ th tap, respectively. From the assumption of the above GBSM, only the complex coefficient for the first tap $(i=1)$ of the $T_{p}-R_{q}$ link contains the LoS component and SB components, which can be expressed as the superposition of $h_{p q}(t, \tau)$ and $h_{1, p q}(t)$

$$
h_{1, p q}(t)=h_{1, p q}^{\mathrm{LoS}}(t)+h_{1, p q}^{\mathrm{SB}}(t)
$$

where

$$
\begin{gathered}
h_{1, p q}^{\mathrm{LOS}}(t)=\sqrt{\frac{K_{p q} \Omega_{1, p q}}{K_{p q}+1}} e^{-j 2 \pi f_{c} \tau_{p q}(t)} e^{j 2 \pi f_{\max } t \cos \left(\phi_{T_{p}}^{\mathrm{LoS}}(t)-\gamma_{R}\right)} \\
h_{1, p q}^{\mathrm{SB}}(t)=\sqrt{\frac{\Omega_{1, p q}}{K_{p q}+1}} \lim _{N_{1} \rightarrow \infty} \sum_{n_{1}=1}^{N_{1}} \frac{1}{\sqrt{N_{1}}} e^{j\left(\psi_{n_{1}}-2 \pi f_{c} \tau_{p q, n_{1}}(t)\right)} \\
\times e^{j 2 \pi f_{\max } t \cos \left(\phi_{R}^{\left(n_{1}\right)}(t)-\gamma_{R}\right)}
\end{gathered}
$$

For other taps $(1<i \leq I)$, their complex tap coefficients of the link of $T_{p}-R_{q}$, contains only SB component, which can be expressed as

$$
\begin{aligned}
& h_{i, p q}(t)=h_{i, p q}^{\mathrm{SB}}(t)=\sqrt{\Omega_{i, p q}} \lim _{N_{i} \rightarrow \infty} \sum_{n_{i}=1}^{N_{i}} \frac{1}{\sqrt{N_{i}}} \\
& \times e^{j\left(\psi_{n_{i}}-2 \pi f_{c} \tau_{p q, n_{i}}(t)\right)} e^{j 2 \pi f_{\max } t \cos \left(\phi_{R}^{\left(n_{i}\right)}(t)-\gamma_{R}\right)}, 1<i \leq I .
\end{aligned}
$$

This channel model is designed to represent the non-stationary behaviour of HST scenarios. Thus in (5a), (5b), and (6), timevarying parameters $\tau_{p q}(t), \phi_{T_{p}}^{\mathrm{LoS}}(t), \tau_{p q, n_{i}}(t)(i=1, \ldots, I)$, and $\phi_{R}^{\left(n_{i}\right)}(t)$ are used, which results in non-stationary characteristics of the underlying GBSM.

In (5a), (5b), and (6), $\Omega_{i, p q}$ denotes the mean power for the $i$ th tap, $\tau_{p q}(t)=\varepsilon_{p q}(t) / c$, and $\tau_{p q, n_{i}}(t)=\left(\varepsilon_{p n_{i}}(t)+\varepsilon_{n_{i} q}(t)\right) / c$ are propagation times of the waves travel through the links $T_{p}-R_{q}$ and $T_{p}-s^{\left(n_{i}\right)}-R_{q}$, respectively, as shown in Fig. 3 . Here, $c$ is the speed of light and $K_{p q}$ is the Ricean factor which indicates the ratio of the power of the LoS component over non-LoS components. The phases $\psi_{n_{1}}$ and $\psi_{n_{i}}$ are assumed as independent and identically distributed (i.i.d.) random variables with uniform distributions over $[-\pi, \pi)$, and

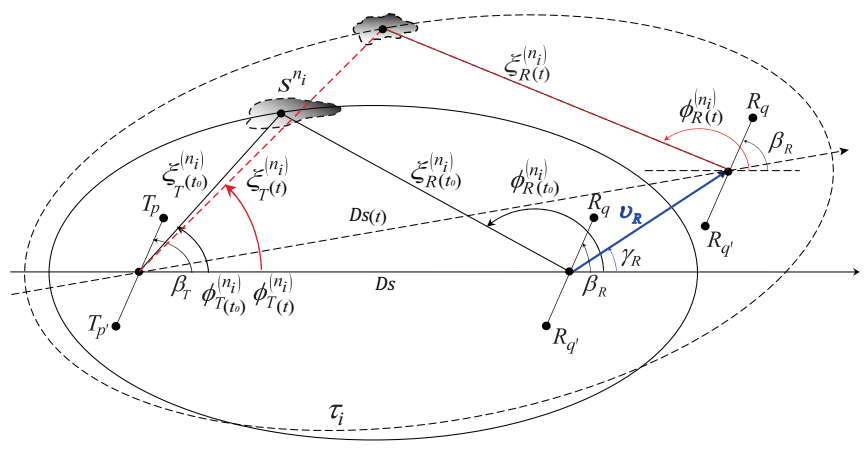

Fig. 4. The time-varying angular parameters in the HST channel model [34].

$f_{\max }$ is the maximum Doppler shift which is related to the carrier frequency and the speed of HST.

In Fig. 4, the time-varying behavior of the GBSM is presented, where the MRS is assumed to move with the speed of $v_{R}$ in the direction defined by the angle of motion $\gamma_{R}$. Further detailed information of calculating all time-varying parameters can be obtained from [33].

In order to make our channel model more accurate and realistic, measurement results (power-delay profile) presented in [28] are used to configure the channel model. In [28], based on measurement results, the authors further classified the HST channel into 5 sub-scenarios in terms of the relative position of the MRS and the BS. In this paper, two typical scenarios are further investigated. They are the Close Area (CA) scenario, which contains the maximum number of taps, and the Closer Area (CEA) scenario, which involves time-varying K-factors. For the CA scenario, $D(t) \in(250,1550] \mathrm{m}$, and $D\left(t_{0}\right)=$ $800 \mathrm{~m}$ is selected as the initial value, $K_{p q}=5.9$. For the CEA scenario, $D(t) \in(20,250] \mathrm{m}$ (we select $D\left(t_{0}\right)=135 \mathrm{~m}$ as the initial value), $K_{p q}$ is time-varying which depends on $D(\mathrm{t})$, and can be expressed as $K_{p q}(t)=-0.0337 D(t)+23.05$, within the range of $[16.31,22.376)$. For both of these two scenarios, distances between different confocal-ellipses $a_{i}(t)$ are calculated refereeing the power-delay profile repetitively.

\section{A. The STCF of the HST Channel Model}

There are various statistical properties of the considered channel model. However, in this paper, only the STCF property is considered as it indicates the spatial correlation among subchannels, and this correlation has significant impact on the 
performance of SM systems. The STCF between two arbitrary channel impulse responses $h_{p q}(t, \tau)$ and $h_{p^{\prime} q^{\prime}}(t, \tau)$ can be defined as:

$$
R_{h}\left(t, \Delta x_{T}, \Delta x_{R}, \Delta t\right)=\mathrm{E}\left\{h_{p q}(t) h_{p^{\prime} q^{\prime}}^{*}(t-\Delta t)\right\}
$$

For this wideband MIMO HST channel, since it assumes uncorrelated scattering, there is no correlation between the underlying processes in different taps. Thus, the over-all STCF is determined by the correlation properties of $h_{i, p q}(t)$ and $h_{i, p^{\prime} q^{\prime}}(t)$ in each tap, and expressed as the superposition of STCF of each taps:

$$
R_{h}\left(t, \Delta x_{T}, \Delta x_{R}, \Delta t\right)=\sum_{i=1}^{I} \Omega_{i, p q} R_{h_{i}}\left(t, \Delta x_{T}, \Delta x_{R}, \Delta t\right)
$$

For the first tap, as it includes both LoS and SB propagation case, its STCF $R_{h_{1}}$ should contain both components, which can be expressed as:

$$
\begin{aligned}
& R_{h_{1}}\left(t, \Delta x_{T}, \Delta x_{R}, \Delta t\right)= \\
& \sqrt{\frac{K_{p q} K_{p^{\prime} q^{\prime}}}{\left(K_{p q}+1\right)\left(K_{p^{\prime} q^{\prime}}+1\right)}} R_{h}^{\mathrm{LoS}}\left(t, \Delta x_{T}, \Delta x_{R}, \Delta t\right) \\
& +\sqrt{\frac{1}{\left(K_{p q}+1\right)\left(K_{p^{\prime} q^{\prime}}+1\right)}} R_{h}^{\mathrm{SB}_{1}}\left(t, \Delta x_{T}, \Delta x_{R}, \Delta t\right)
\end{aligned}
$$

For remaining taps, as they only contain SB components, the STCF $R_{h_{i}}(i=2 \ldots I)$ is only determined by the STCF of SB component which can be expressed as:

$$
R_{h_{i}}\left(t, \Delta x_{T}, \Delta x_{R}, \Delta t\right)=R_{h}^{\mathrm{SB}_{i}}\left(t, \Delta x_{T}, \Delta x_{R}, \Delta t\right),(i=2 \ldots I) .
$$

- In the case of the LoS component,

$$
\begin{aligned}
R_{h}^{\mathrm{LoS}}\left(t, \Delta x_{T}, \Delta x_{R}, \Delta t\right) & =e^{j 2 \pi\left[P \cos \beta_{T}-Q \cos \left(\phi_{T_{p}}^{\mathrm{LoS}}(t)-\beta_{R}\right)\right]} \\
& \times e^{j 2 \pi f_{\max } \cos \left(\phi_{T_{p}}^{\mathrm{LoS}}(t-\Delta t)-\gamma_{R}\right) \Delta t}
\end{aligned}
$$

where $P=\left(p^{\prime}-p\right) \Delta x_{T} / \lambda, Q=\left(q^{\prime}-q\right) \Delta x_{R} / \lambda$,

- In the case of the SB component, $i=1 \ldots I$

$$
\begin{aligned}
& R_{h}^{\mathrm{SB}}\left(t, \Delta x_{T}, \Delta x_{R}, \Delta t\right)=\frac{1}{2 \pi I_{0}\left(k_{R}^{(i)}\right) \sqrt{K_{p q} K_{p^{\prime} q^{\prime}}}} \\
\times & \int_{-\pi}^{\pi} e^{k_{R}^{(i)} \cos \left(\phi_{R}^{(i)}-\mu_{R}^{(i)}(t)\right)} e^{j 2 \pi\left[P \cos \left(\phi_{T}^{(i)}-\beta_{T}\right)+Q \cos \left(\phi_{R}^{(i)}-\beta_{R}\right)\right]} \\
\times & e^{j 2 \pi \xi_{T R}^{\left(n_{i}\right)}(t, \Delta t)} e^{j 2 \pi f_{\max } \cos \left(\phi_{R}^{(i)}-\gamma_{R}\right) \Delta t} \mathrm{~d} \phi_{R}^{(i)}
\end{aligned}
$$

where $\xi_{T R}^{n_{i}}(t, \Delta t)=\xi_{T}^{n_{i}}(t-\Delta t)-\xi_{T}^{n_{i}}(t)+\xi_{R}^{n_{i}}(t-\Delta t)-$ $\xi_{R}^{n_{i}}(t)$

\section{B. Stationary interval in terms of STCF}

For this HST channel model, its unique characteristic is the non-stationary behavior. As conventional statistical properties are not able to accurately describe this behavior, it is necessary to introduce some new metrics. In [30], a property named the stationary interval was proposed, which was calculated in terms of local region of stationarity to describe the nonstationary characteristic of the HST scenario. In our system, as the BER performance is directly affected by the STCF, we will propose to the stationary interval in terms of the STCF.

This new statistic property is defined as the maximum time duration within which the required similarity of the STCF behavior can be maintained. Mathematically, we can describe the stationary interval as the time duration that makes the autocorrelation function (ACF) of the STCF maintains no less than a given threshold, i.e.,

$$
\begin{aligned}
t_{\text {int }}=\max \left\{t_{\text {int }} \mid \operatorname{ACF}(\right. & R_{h}\left(t, \Delta x_{T}, \Delta x_{R}\right), \\
& \left.\left.R_{h}\left(t+t_{\text {int }}, \Delta x_{T}, \Delta x_{R}\right)\right) \leqslant C_{\text {threshold }}\right\}
\end{aligned}
$$

Here, the ACF of the STCF is expressed as

$$
\begin{gathered}
\operatorname{ACF}\left(R_{h}\left(t, \Delta x_{T}, \Delta x_{R}\right), R_{h}\left(t+t_{\text {int }}, \Delta x_{T}, \Delta x_{R}\right)\right) \\
=\operatorname{E}\left\{R_{h}\left(t, \Delta x_{T}, \Delta x_{R}\right) R_{h}^{*}\left(t+t_{\text {int }}, \Delta x_{T}, \Delta x_{R}\right)\right\}
\end{gathered}
$$

which describes how the STCF is correlated at different time instants with the time interval $t_{\text {int }}$. In Section $\mathrm{V}$, we will further investigate how this property affects the BER performance of SM systems.

\section{Channel estimation error model}

In a real communication system, the estimated channel coefficient at the Rx is used to recover the transmitted signal. The estimated channel coefficient always comes with estimation errors. In order to study how estimation errors affect the system performance, accurate error models should be used. Research works regarding estimated error model have been reported in [38] and [39]. However, these error models cannot be directly used in our system as they are only valid to narrowband channels. In this paper, a novel estimation error scheme for the wideband HST channel model is presented. In this model, errors caused by the estimation delay are represented by considering a decline factor and an error vector. As the channel is a wideband one, the final error model is the sum of errors for each tap. The error model is expressed as

$$
\begin{aligned}
H_{\text {est }}\left(t, \tau_{\text {est }}\right) & =\sum_{i=1}^{I} \rho_{i}\left(t+\tau_{\text {est }}\right) H_{i,}(t) \\
& +\sum_{i=1}^{I} \Omega_{i}\left(1-\rho_{i}\left(t+\tau_{\text {est }}\right)\right) \cdot \varepsilon_{\text {est }} .
\end{aligned}
$$

In (15), $\rho_{i}\left(t+\tau_{\text {est }}\right)$ is the ACF for the ith tap of the channel at time $t$ with the estimation delay $\tau_{\text {est }}$, which is used as the decline factor, and $\varepsilon_{\text {est }}$ is the random error vector which is assumed to obey the unit-variance zero-mean complex Gaussian distribution.

\section{TIME-VARYING THEORETICAL BER ANALYSIS}

By using the union bound method mentioned in [36], [40]-[43], the theoretical average bit error rate (ABER) of SM systems can be calculated. In this paper, the improved upper-bound method presented in [36] is used, which further classifies errors into 3 categories: 1) error only occurs in the 
digital modulation part with the probability $\underset{\text { Signal }}{\mathrm{ABER}}$ 2) error only occurs in spatial modulation part with the probability ABER and 3) error occurs in both parts with the probability Spatial

Aoint. The overall upper-bound expression of the BER is the combination of these three parts. In this paper, as the channel is time-varying, the theoretical derivation should also consider to be time-varying, which is expressed as:

$$
\underset{\mathrm{SM}}{\operatorname{ABER}}(t) \leqslant \underset{\text { Signal }}{\operatorname{ABER}}(t)+\underset{\text { Spatial }}{\operatorname{ABER}}(t)+\underset{\text { Joint }}{\operatorname{ABER}}(t)
$$

$\underset{\operatorname{Signal}}{\operatorname{ABER}}(t)$ represents the case that at time $t$ error only occurs in the digital modulation part while the transmit antenna index is correctly detected. It is similar to the single-input-multipleoutput (SIMO) case. The BER expression is given by

$$
\underset{\text { Signal }}{\operatorname{ABER}}(t)=\frac{1}{N_{t}} \frac{\log _{2}(M)}{m} \sum_{n_{t}=1}^{N_{t}} \underset{\mathrm{MOD}}{\operatorname{APEB}}\left(t, n_{t}\right),
$$

and

$$
\underset{\operatorname{MOD}}{\operatorname{APEB}}\left(t, n_{t}\right)=\frac{1}{M} \frac{1}{\log _{2}(M)} N_{H}(\chi \rightarrow \tilde{\chi}) P(t, \chi \rightarrow \tilde{\chi}) .
$$

In (18), $N_{H}(\chi \rightarrow \tilde{\chi})$ is the Hamming distance between modulated symbols $\chi$ and $\tilde{\chi}, P(t, \chi \rightarrow \tilde{\chi})$ denotes the pairwise error probability (PEP) that the transmitted symbol $\chi$ is detected as $\tilde{\chi}$ at time $t$. In (17) and (18), A ABER is determined by the digital modulation scheme. For example, if the M-PSK modulation scheme is used, (18) can be derived out referring [36], [40] as

$$
\begin{aligned}
\underset{\operatorname{MOD}}{\operatorname{APEB}\left(t, n_{t}\right)} & =\frac{1}{\log _{2}(M)} \sum_{l=1}^{M-1}\left[\left(2\left|\frac{l}{M}-\left\lfloor\frac{l}{M}\right\rfloor\right|\right.\right. \\
& \left.\left.+2 \sum_{k=2}^{\log _{2}(M)}\left|\frac{l}{2^{k}}-\left\lfloor\frac{l}{2^{k}}\right\rfloor\right|\right)\right] P_{l}\left(t, n_{t}\right),
\end{aligned}
$$

where:

$$
\begin{aligned}
P_{l}\left(t, n_{t}\right) & =\frac{1}{2 \pi} \int_{0}^{\pi[1-(2 l-1) / M]} T_{l}^{-}\left(\theta, n_{t}, t\right) d \theta \\
& -\frac{1}{2 \pi} \int_{0}^{\pi[1-(2 l+1) / M]} T_{l}^{+}\left(\theta, n_{t}, t\right) d \theta,
\end{aligned}
$$

and:

$$
\begin{aligned}
& T_{l}^{-}\left(\theta, n_{t}, t\right)=M_{r}\left(2 \gamma \frac{\sin ^{2}[\pi(2 l-1) / M]}{\sin ^{2} \theta}, t\right), \\
& T_{l}^{+}\left(\theta, n_{t}, t\right)=M_{r}\left(2 \gamma \frac{\sin ^{2}[\pi(2 l+1) / M]}{\sin ^{2} \theta}, t\right)
\end{aligned}
$$

In (21), $\gamma$ is the signal-to-interference-plus-noise ratio (SINR). Under the condition with channel estimation error, the SINR can be defined as:

$$
\gamma=\frac{E_{m} \cdot \sum_{i=1}^{I} \rho_{i}\left(t+\tau_{e s t}\right) \Omega_{i}}{N+I+\sum_{i=1}^{I} \Omega_{i}\left(1-\rho_{i}\left(t+\tau_{\text {est }}\right)\right)}
$$

where $E_{m}$ is the transmit power, $N$ is the noise power, $I$ is the power of ISI. $M_{r}(., t)$ is the time-varying moment generation function (MGF) of $\sum_{n_{r}=1}^{N_{R}}\left\|h_{n_{t}, n_{r}, e s t}(t) e\right\|^{2}$, where $e=\chi-\tilde{\chi}$. The close form expression of $M_{r}(., t)$ is

$$
M_{r}(s, t)=\prod_{i=1}^{N_{R}}\left(1-s \lambda_{i}\left(t+\tau_{e s t}\right) e\right)^{-1}
$$

In (23), $\lambda_{i}(t)$ is the eigenvalue of time-varying $\mathrm{Rx}$ correlation indicator matrix $R_{R x}(t)$. Similarly, we can define $R_{T x}(t)$ as the time-varying correlation indicator matrix of Tx. Considering the this GBSM channel model and referring [44], these two matrices are expressed as

$R_{T x}(p, \tilde{p})=R_{h}\left\{h_{q, p} h_{q, \tilde{p}}\right\}(t), R_{R x}(q, \tilde{q})=R_{h}\left\{h_{q, p} h_{\tilde{q}, p}\right\}(t)$,

where $R_{h}\left\{h_{q, p} h_{q, \tilde{p}}\right\}(t)$ is the STCF of the HST channel model at time $t$, which indicates the correlation between $h_{q, p}$ and $h_{q, \tilde{p}}$.

For the case that error only occurs in the spatial modulation part, which is expressed as $\underset{\text { Spatial }}{\mathrm{ABER}}$ previously, we can refer to related investigations on space shift keying (SSK) systems, [41], [42]. This part can be expressed as

$$
\underset{\text { Spatial }}{\operatorname{ABER}}(t)=\frac{1}{M} \frac{\log _{2}\left(N_{t}\right)}{m} \sum_{l=1}^{M} \underset{\mathrm{SSK}}{\operatorname{APEB}}(t, l) .
$$

APEB can be further expressed as

$$
\begin{aligned}
\underset{\mathrm{SSK}}{\operatorname{APEB}}(t, l) & =\frac{1}{N_{t}} \frac{1}{\log _{2}\left(N_{t}\right)} \\
& \sum_{n_{t}=1}^{N_{t}} \sum_{\tilde{n}_{t}=1}^{N_{t}}\left[N_{H}\left(n_{t} \rightarrow \tilde{n}_{t}\right) P\left(t, \kappa_{l}, n_{t} \rightarrow \tilde{n}_{t}\right)\right],
\end{aligned}
$$

where $N_{H}\left(n_{t} \rightarrow \tilde{n}_{t}\right)$ is the Hamming distance between antenna indices $n_{t}$ and $\tilde{n}_{t}$, the PEP that at time $t$ is represented as $P\left(t, \kappa_{l}, n_{t} \rightarrow \tilde{n}_{t}\right)$, the active antenna $\tilde{n}_{t}$ is detected given that $n_{t}$ was transmitted with the modulation modulus $\kappa_{l}$. If constant modulus modulation schemes such as M-PSK are used, $\kappa_{l}$ is a constant value as $\kappa$ and (25) can be simplified as

$$
\begin{aligned}
\underset{\text { Spatial }}{\operatorname{ABER}}(t) & =\frac{1}{N_{t} m} \sum_{n_{t}=1}^{N_{t}} \sum_{\tilde{n}_{t}=1}^{N_{t}}\left[N_{H}\left(n_{t} \rightarrow \tilde{n}_{t}\right)\right. \\
& \left.\times \frac{1}{\pi} \int_{0}^{\pi / 2} M_{n_{t}, \tilde{n}}\left(\gamma \frac{k^{2}}{2 \sin ^{2} \theta}, t\right) d \theta\right] .
\end{aligned}
$$

In (27), $M_{n_{t}, \tilde{n}_{t}}(., t)$ is the MGF of $\sum_{\substack{n_{r}=1 \\ \text { as: }}}^{N_{R}}\left\|h_{n_{t}, n_{r}, \text { est }}(t)-h_{\tilde{n}_{t}, n_{r}, \text { est }}(t)\right\|^{2}$, which can be expressed

$$
M_{n_{t}, \tilde{n}_{t}}(s, t)=\prod_{j=1}^{N_{T}} \prod_{i=1}^{N_{R}}\left(1-s \lambda_{i}\left(t+\tau_{e s t}\right) u_{j}\left(t+\tau_{e s t}\right)^{-1}\right),
$$

where $u_{j}(t)$ is the eigenvalue of receivers correlation indicator matrix $R_{T x}$ 


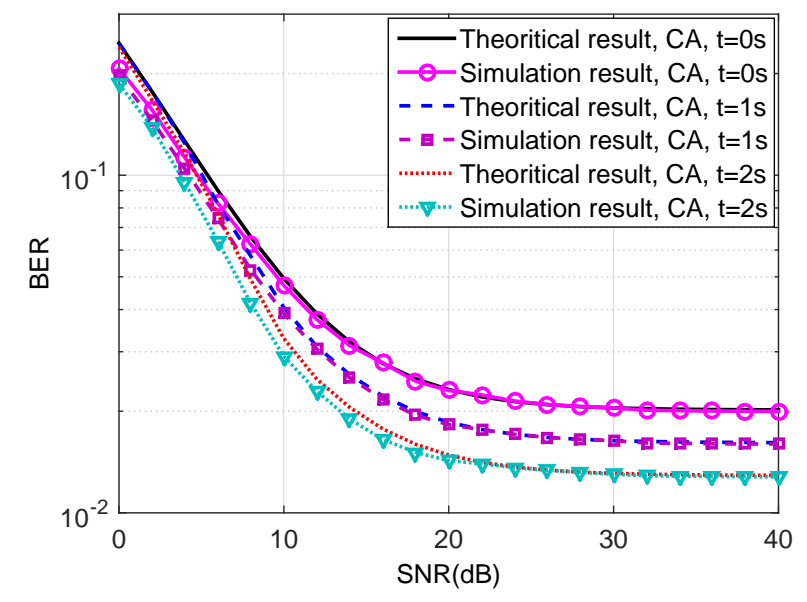

Fig. 5. Theoretical and simulated BER performance of the SM system under the CA scenario at different time instants.

For the third case, $\underset{\text { Joint }}{\mathrm{ABER}}$, both digital modulation and spatial modulation parts are incorrect. It can be expressed as:

$$
\begin{aligned}
\underset{\text { Joint }}{\operatorname{ABER}}(t) & =\frac{1}{N_{t} m} \sum_{n_{t}=1}^{N_{t}} \sum_{\tilde{n}_{t} \neq n_{t}=1}^{N_{t}}\left[N_{H}\left(n_{t}, \chi \rightarrow \tilde{n}_{t}, \tilde{\chi}\right)\right. \\
& \left.\times P\left(t, n_{t}, \chi \rightarrow \tilde{n}_{t}, \tilde{\chi}\right)\right],
\end{aligned}
$$

where $N_{H}\left(\left(n_{t}, \chi\right) \rightarrow\left(\tilde{n}_{t}, \tilde{\chi}\right)\right)$ is the Hamming distance for the error case that symbol $\chi$ transmitted by antenna $n_{t}$ is detected as $\tilde{\chi}$ sent by antenna $\tilde{n}_{t}$, and $P\left(t,\left(n_{t}, \chi\right) \rightarrow\left(\tilde{n}_{t}, \tilde{\chi}\right)\right)$ is the corresponding PEP which can be represented as

$$
P\left(t,\left(n_{t}, \chi\right) \rightarrow\left(\tilde{n}_{t}, \tilde{\chi}\right)\right)=\frac{1}{\pi} \int_{0}^{\frac{\pi}{2}} M_{n_{t}, \tilde{n}_{t} \tilde{\chi}}\left(\frac{\gamma}{2 \sin ^{2} \theta},(t)\right) d \theta .
$$

In (30), $\quad M_{n_{t} \chi, \tilde{n}_{t} \tilde{\chi}}(., t) \quad$ is the MGF of $\sum_{n_{r}=1}^{N_{r}}\left|h_{n_{t}, n_{r}, e s t}(t) \chi-h_{\tilde{n}_{t}, n_{r}, e s t}(t) \tilde{\chi}\right|^{2}$, with the expression of $M_{n_{t}, \tilde{n}_{t} \tilde{\chi}}(s, t)=\prod_{j=1}^{N_{T}} \prod_{i=1}^{N_{R}}\left(1-s \lambda_{i}\left(t+\tau_{e s t}\right) e u_{j}\left(t+\tau_{e s t}\right)\right)^{-1}$.

\section{Results And Discussions}

In this section, theoretical derivation results and simulation results are presented to evaluate the performance of SM systems under the non-stationary wideband HST channel model.

\section{A. Simulation settings}

In this paper, all the BER simulation results were obtained using the Monte Carlo simulation method. The BER result was obtained by averaging over 10 simulation runs, with each run transmitting 100000 symbols. The system is assumed to be a 2x2 MIMO LTE system working with $1.4 \mathrm{MHz}$ bandwidth and BPSK modulation scheme. According to [28] and [45], the following key parameters were used to configure all simulations: $f_{c}=2.6 \mathrm{GHz}$ and $v_{R}=300 \mathrm{~km} / \mathrm{h}$ with the movement angle $\gamma_{R}=30^{\circ}$. The corresponding maximum Doppler shift is $f_{\max }=722 \mathrm{~Hz}$.

The initial AoAs $\mu_{R}^{(i)}\left(t_{0}\right)$ were selected based on the D2a moving networks scenario in WINNER II channel model [12].

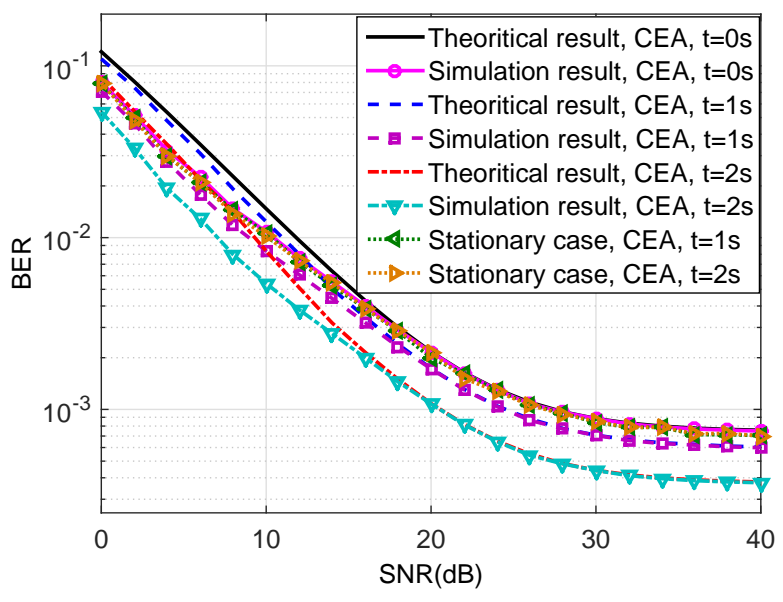

Fig. 6. Theoretical and simulated BER performance of the SM system under the CEA scenario at different time instants.

The concentration parameter of the von Mise distribution was chosen as $k_{R}^{(i)}=6$. The space between two antenna elements of Tx and Rx sides was configured as $0.5 \lambda$ with tilt angles as $\beta_{R}=\beta_{T}=60^{\circ}$. The distance between the BS and the track is defined as $D_{\text {min }}=50 \mathrm{~m}$. For other parameters related to different scenarios, please refer to section III. Please note that in this paper, equalizer was not used in order to purely investigate the effect of the non-stationary wideband HST channel model.

\section{B. Theoretical and simulation results}

In Fig. 5, theoretical results of ABER for SM systems under the non-stationary wideband HST channel model in $\mathrm{CA}$ scenarios are compared with simulation results. As the theoretical result is the upper bound for the BER performance, it will be higher than the simulation results at low SNR range. The comparison, clearly indicates an excellent agreement of the theoretical derivation with simulation results at the high SNR range. We can conclude the theoretical ABER can offer reasonable accuracy and therefore, it can be used to estimate the BER of SM systems under nonstationary wideband channels. From this figure, we can also observe that the BER performance of the SM system shows the time-varying characteristic. When $S N R \geq 20 \mathrm{~dB}$, the BER performance keeps almost the same even with the increase of SNR, which exhibits a error floor behavior. This is because for wideband channels, the received signal also suffers from ISI besides noise. When the transmit power increases, the ISI increases as well and becomes the key factor affecting the BER performance. This is in line with [22], where it stated when the transmit power turns to a much higher level than the noise, the SINR will approach $\frac{E_{m}}{I}$ without considering channel estimation error, which will generate an error floor.

In Fig. 6, similar observations can be obtained. Under the CEA scenario, theoretical results can also match simulation results very well even involved the time-varying $K$ factor. Similar to the CA scenario, at high SNR range, the system's performance also suffers from the ISI. However, for the CEA scenario, due to the ISI level is lower than the CA scenario, 


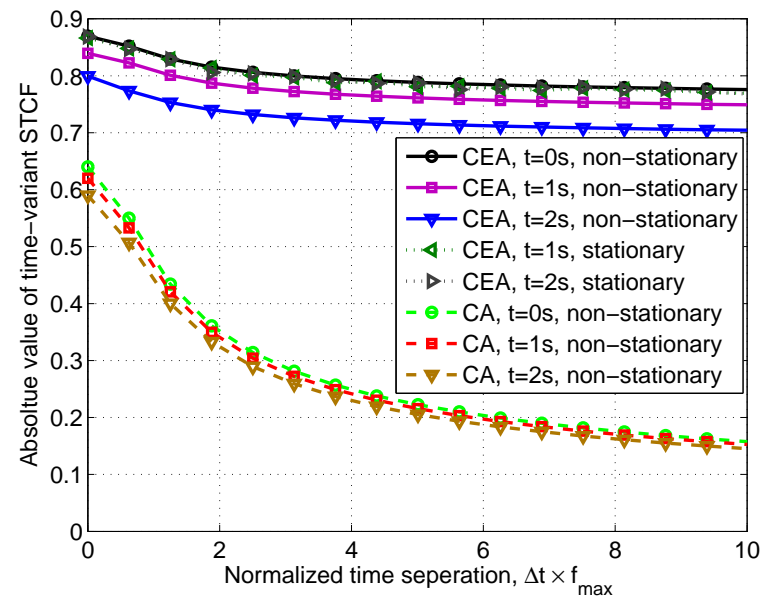

Fig. 7. Absolute values of STCFs for HST channel models with different scenarios.

the SM system can offer better performance than under the CA scenario. The BER performance in the CEA scenario also shows the time-varying characteristic. By comparing with results of the CA scenario, we can find that under the CEA scenario, the time-varying characteristic is much stronger. With the same time change, the BER result of the CEA scenario appears higher change ratio. The reason behind this phenomena is that for the CA scenario, only the distance between the BS and the MRS and AoA are time-varying, but for the CEA scenario, besides these above mentioned parameters, the $K$ factor which has significantly influence on the correlation properties of the channel are time-varying.

In this figure, we also show BER results that when the channel is configured as a stationary stochastic channel model which means all parameters a not time-varying. It can be observed that under the stationary assumption, there is negligible BER changes compared with the non-stationary case. This observation can be proved in Fig. 7 where the STCFs of the channel model is shown. For the CA scenario, the similar results can be obtained. As the CA scenario is more stationary than the CEA scenario even when using timevarying parameters, and also avoid making Fig. 7 messing, we will not repeat the results of the stationary CA condition in this paper.

In Fig. 7, STCFs of the HST channel model under the $\mathrm{CA}$ and the CEA scenario are shown. It can support our previous analysis. Firstly, the time-varying behavior of the BER performance can be further explained. From this figure, we can find that the STCF for both scenarios shows timevarying behavior. However, considering together with the BER figures, it can be observed that the CEA scenario exhibits stronger time-varying behavior than the CA scenario due to the influence of time-varying $K$ factor. Secondly, comparing the STCF of the CA and the CEA scenario, we can find that for the CA scenario, its correlation of sub-channels is much lower than that of the CA scenario due to the higher $K$ factor. In principle, the BER performance of the CA scenario should be better than that of the CEA scenario. However, from Fig. 5 and Fig. 6, it is clear the SM system has superior

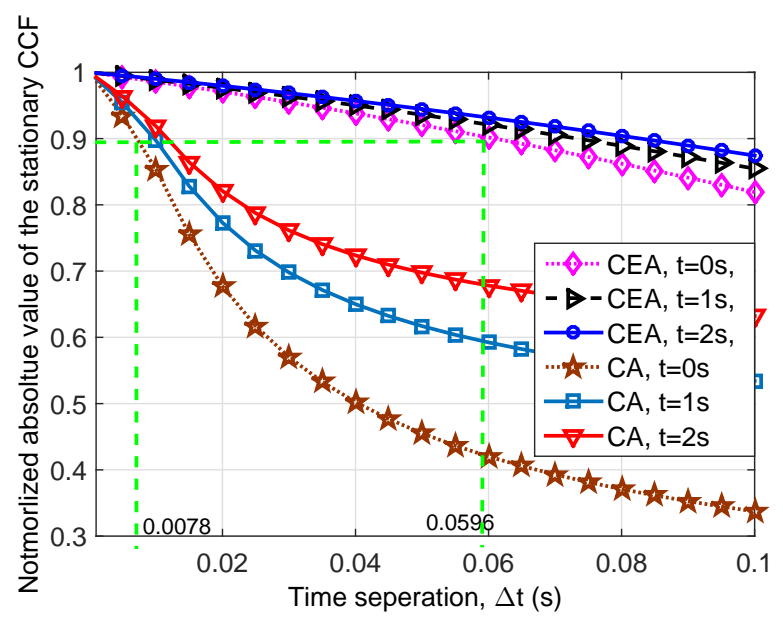

Fig. 8. Stationary interval of the HST channel model under CA and CEA scenarios.

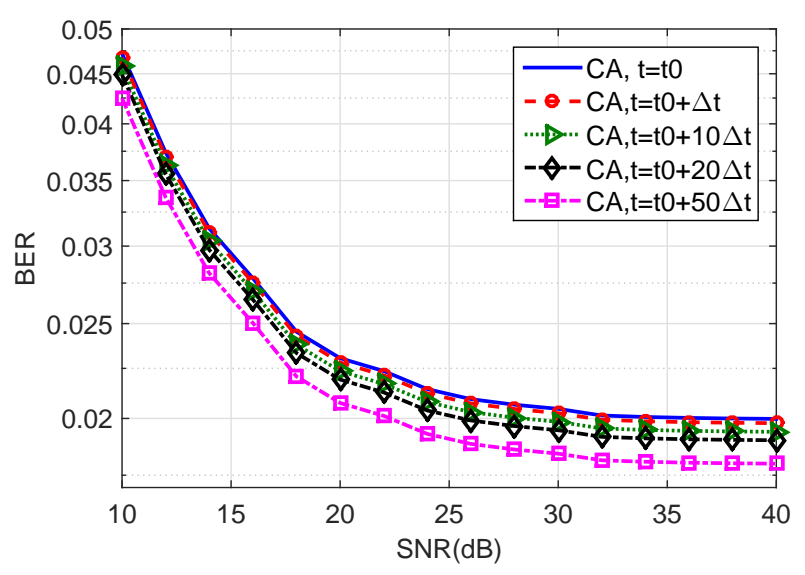

Fig. 9. BER performance of the SM system under the CA scenario in terms of the stationary interval $\left(\Delta t=t_{\text {int }}=0.0596 \mathrm{~s}\right)$.

performance under the CEA scenario. That is because under the CA scenario, the SM system suffers stronger ISI than the CEA scenario. From this comparison, we can conclude that the ISI has much stronger effect to the performance of SM system under wideband channel compared with other issues. Thirdly, under the stationary assumption, the STCF is not change with the time $t$, but depend to the time separation $\Delta t$, which will result in the no time-varying BER performance.

In Fig. 8, the stationary interval $t_{\text {int }}$ in terms of STCF for the non-stationary HST channel model under both the CA and the CEA scenarios are presented. In this work, the similarity threshold $C_{\text {threshold }}=0.9$ is used. From this figure, it can be observed that the CA scenario has the longer stationary interval than the CEA scenario, which can match our previous observations that the CA scenario is relative more stationary than the CEA scenario.

In Fig. 9 and 10, the BER performance are investigated in term of the stationary interval. In our simulation, stationary intervals are selected from the threshold $C_{\text {threshold }}=0.9$. From simulation results, it can be observed that within the stationary interval, due to the correlation between sub-channels maintains relatively stationary, the BER performance can also keeps the stable behavior. However, along with time difference increases, due to the correlation property falling below the nec- 


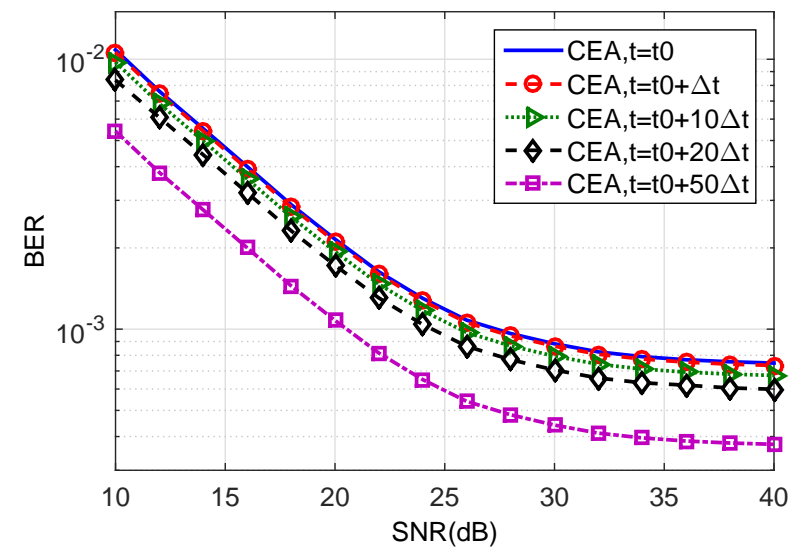

Fig. 10. BER performance of the SM system under the CEA scenario in terms of the stationary interval $\left(\Delta t=t_{\text {int }}=0.0078 \mathrm{~s}\right)$.

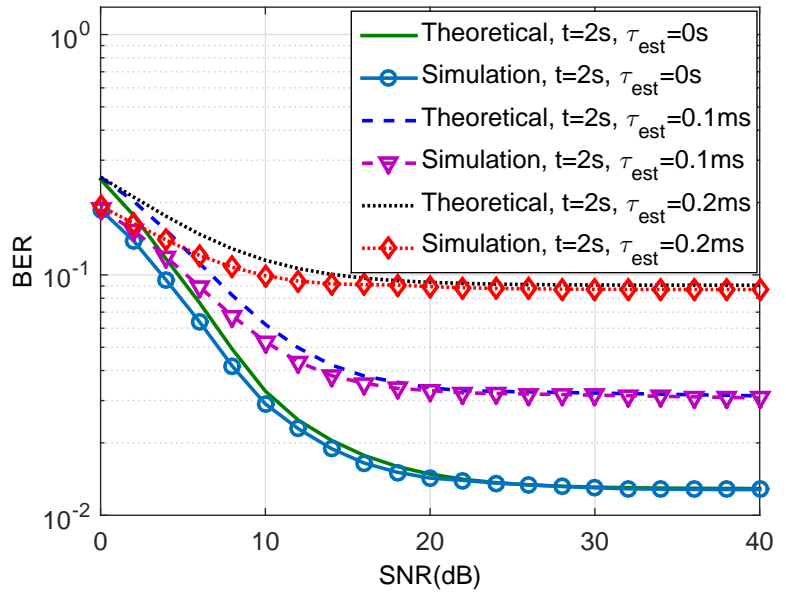

Fig. 11. BER performance of the SM system under the CA scenario in terms of different estimation delays $\left(f_{\max }=722 \mathrm{~Hz}\right)$.

essary threshold, the behavior on BER performance changes. From this investigation, we can observe the importance of this statistic property to the system as it can be used as a guideline for selecting the channel estimation intervals.

In Fig. 11 and Fig. 12, the BER performance of SM system are investigated under the CA scenario with non-ideal channel estimation, impacts of channel estimation delay and Doppler frequency are considered separately. It can be observed that theoretical upper bounds and simulation results can match very well for all cases. The accuracy and the flexibility of our theoretical BER framework can be noticed, it can capture the BER behavior of SM system under the complex HST propagation scenarios. In Fig.11, the BER performance is investigated with different estimation delay while under the case of fixed Doppler frequency $\left(f_{\max }=722 \mathrm{~Hz}\right)$. It can be observed that better BER performance can be achieved with smaller channel estimation delays. This predominance can be explained by Fig. 13 which shows that with the fixed Doppler frequency, the shorter time difference (also refers to channel estimation delay) will result in the better coherence between the estimated channel and the real channel.

In Fig.12, the BER performance of SM with the fixed estimation delay $(\tau=0.2 \mathrm{~ms})$ but different Doppler frequencies

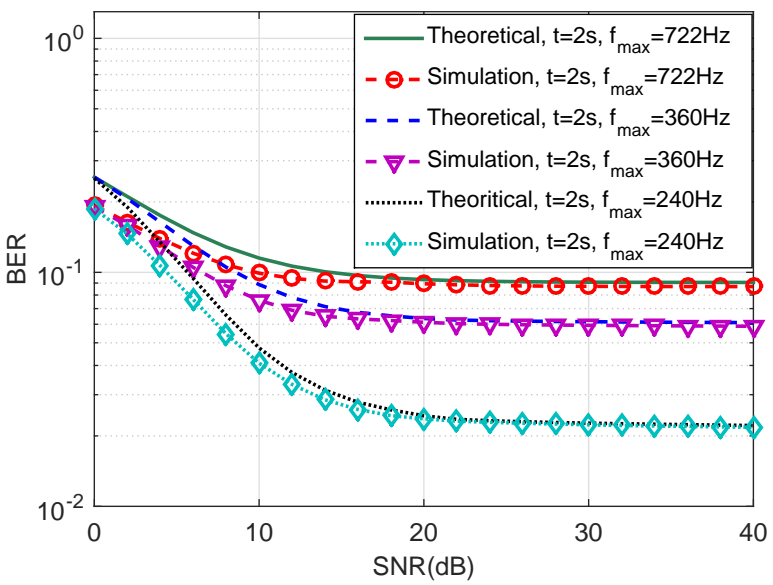

Fig. 12. BER performance of the SM system under the CA scenario in terms of different Donnler freauencies $\left(\tau_{o \circ}=0.2 \mathrm{~ms}\right)$.

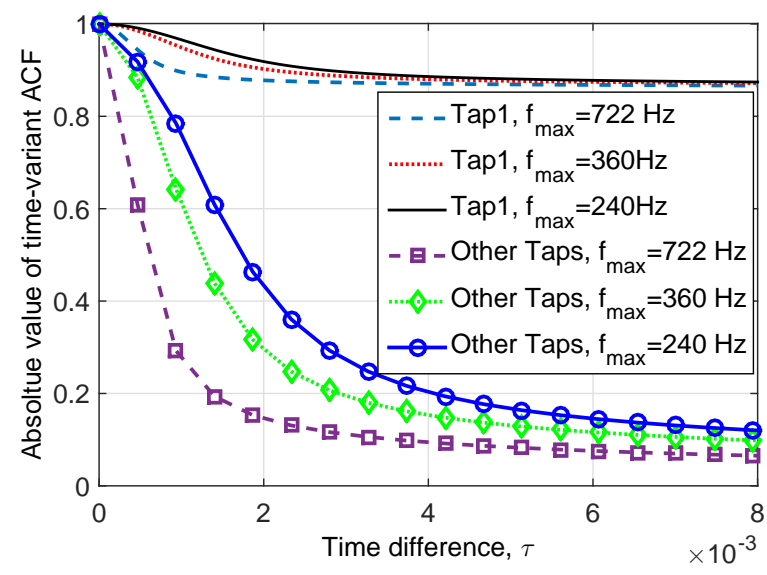

Fig. 13. The absolute values of the time-variant ACF of different Taps of the HST channel model with different Doppler frequency at the time instant of $t=2 \mathrm{~s}$.

are illustrated. It can be found that with fixed estimation delays, the higher Doppler frequency which corresponding to the higher speed will cause poor BER performance. That is because higher Doppler frequency will shorten the coherence time of the channel, and will significantly decrease the reliability of SM systems.

\section{Conclusions}

In this paper, we have investigated the BER performance of SM systems under a novel non-stationary wideband HST channel model with different propagation scenarios. Measurement results have been considered which make the channel model more accurate to capture the reality of HST communication scenarios. Accurate theoretical BER expression of SM systems under the non-stationary wideband HST channel model with non-ideal channel estimation has been derived. The influence of different time-varying parameters on the BER performance of SM systems have comprehensively been investigated. Additionally, a novel statistic property called stationary interval has been proposed to be calculated in terms of the STCF in order to accurately capture the non-stationary behavior of the channel. Its impact on the BER performance of SM systems has also been investigated. From theoretical and 
simulation results, we can observe that the BER performance of SM systems shows a time-varying behavior due to the nonstationary HST channel model. The BER performance of SM systems under the HST channel model is mainly affected by the correlation between sub-channels, ISI, Doppler shift, and channel estimation errors.

\section{APPENDIX A}

DERIVATION OF (27)

For the case that error only occurs in the spatial modulation part, referring to the ML detector algorithm in (3), this error can only occurs when $\left\|y-h_{n_{t}, n_{r}, e s t}(t) \kappa_{l}\right\|^{2}>$ $\left\|y-h_{\tilde{n}_{t}, n_{r}, e s t}(t) \kappa_{l}\right\|^{2}$. Thus, $P\left(t, \kappa_{l}, n_{t} \rightarrow \tilde{n}_{t}\right)$ can be written as

$$
\begin{aligned}
& P\left(t, \kappa_{l}, n_{t} \rightarrow \tilde{n}_{t}\right) \\
& \quad=P\left(\left\|y-h_{n_{t}, n_{r}, e s t}(t) \kappa_{l}\right\|^{2}>\left\|y-h_{\tilde{n}_{t}, n_{r}, e s t}(t) \kappa_{l}\right\|^{2}\right) \\
& \quad=Q\left(\gamma\left\|h_{n_{t}, n_{r}, e s t}(t) \kappa_{l}-h_{n_{t}, n_{r}, e s t}(t) \kappa_{l}\right\|^{2}\right),
\end{aligned}
$$

where $Q($.$) is the \mathrm{Q}$-function. Based on the alternative integral expression of Q-function and MGF-based approach [40], [43], $P\left(t, \kappa_{l}, n_{t} \rightarrow \tilde{n}_{t}\right)$ can be written as

$$
P\left(t, \kappa_{l}, n_{t} \rightarrow \tilde{n}_{t}\right)=\frac{1}{\pi} \int_{0}^{\pi / 2} M_{n_{t}, \tilde{n}_{t}}\left(\gamma \frac{\kappa_{l}^{2}}{2 \sin ^{2} \theta}, t\right) d \theta .
$$

Thus,

$$
\begin{aligned}
& \underset{\text { Spatial }}{\operatorname{ABER}}(t)=\frac{1}{M} \frac{\log _{2}\left(N_{t}\right)}{m} \\
& \sum_{l=1}^{M} \frac{1}{N_{t}} \frac{1}{\log _{2}\left(N_{t}\right)} \sum_{n_{t}=1}^{N_{t}} \sum_{\tilde{n}_{t}=1}^{N_{t}}\left[N_{H}\left(n_{t} \rightarrow \tilde{n}_{t}\right) \frac{1}{\pi} \int_{0}^{\pi / 2} M_{n_{t}, \tilde{n}_{t}}\left(\gamma \frac{\kappa_{l}^{2}}{2 \sin ^{2} \theta}, t\right) d \theta\right] .
\end{aligned}
$$

\section{REFERENCES}

[1] D. Xin. Record-breaking train on track. [Online]. Available: http://www.chinadaily.com.cn/china/2010-12/04/content-11651930.html, 2010.

[2] D. T. Fokum and V. S. Frost, "A survey on methods for broadband internet access on trains," IEEE Commun. Surveys \& Tutotials, vol. 12, no. 2, pp. 171-185, 2nd Quarter 2010.

[3] C.-X. Wang, S. Wu, L. Bai, X. You, J. Wang, and C.-L. I, "Recent advances and future challenges for massive MIMO channel measurements and models," Sci. China Inf. Sci., Invited Paper, vol. 59, no. 2, pp. 1-16, Feb. 2016.

[4] C.-X. Wang, F. Haider, X. Gao, X.-H. You, Y. Yang, D. Yuan, H. Aggoune, H. Haas, S. Fletcher, and E. Hepsaydir, "Cellular architecture and key technologies for 5G wireless communication networks," IEEE Commun. Mag., vol. 52, no. 2, pp. 122-130, Feb. 2014.

[5] Y. Zhou, Z. Pan, J. Hu, J. Shi, and X. Mo, "Broadband wireless communications on high speed trains," in Proc. IEEE WOCC'11, New Jersey, USA, Apr. 2011.

[6] J. Wang, H. Zhu, and N. J. Gomes, "Distributed antenna systems for mobile communications in high speed trains," IEEE J. Sel. Areas Commun., vol. 30, no. 4, pp. 675-683, May 2012.

[7] C. Yang, L. Lu, C. Di, and X. Fang, "An on-vehicle dual-antenna handover scheme for high-speed railway distributed antenna system," in Proc. IEEE WiCOM'10, Chengdu, China, Sept. 2010, pp. 1-4.

[8] H. Wei, D. Wang, J. Wang, and X. You,, "Impact of RF mismatches on the performance of massive MIMO systems with ZF precoding," Sci. China Inf. Sci., Invited Paper, vol. 59, no. 2, pp. 26-39, Feb. 2016.

[9] L. Tian, J. Li, Y. Huang, J. Shi, and J. Zhou, "Seamless dual-link handover scheme in broadband wireless communication systems for high-speed rail," IEEE J. Sel. Areas Commun., vol. 30, no. 4, pp. 708-718, May 2012.

[10] O. B. Karimi, L. J. Liu, and C. Wang, "Seamless wireless connectivity for multimedia services in high speed trains," IEEE J. Sel. Areas Commun., vol. 30, no. 4, pp. 729-739, May 2012.
[11] F. Haider, C.-X. Wang, B. Ai, H. Haas, and E. Hepsaydir, "Spectralenergy efficiency trade-off of cellular systems with mobile femtocell deployment," in IEEE Trans. Veh. Technol., 2016, in press.

[12] P. Kyösti, et al., "WINNER II channel models," IST-4-027756, WINNER II D1.1.2, v1.2, Apr. 2008.

[13] "Guidelines for evaluation of radio interface technologies for IMTadvanced," ITU, Geneva, Switzerland, Tech. Rep. ITU-R M.2135-1, Dec. 2009.

[14] R. Mesleh, H. Haas, S. Sinanovic, C. W. Ahn, and S. Yun, "Spatial modulation," IEEE Trans. Veh. Technol., vol. 57, no. 4, pp. 2228-2241, July. 2008.

[15] J. Jeganathan, A. Ghrayeb, and L. Szczecinski, "Spatial modulation: optimal detection and performance analysis," IEEE Commun. Lett., vol. 12, no. 8, pp. 545-547, Aug. 2008.

[16] J. Jeganathan, A. Ghrayeb, L. Szczecinski, and A. Ceron, "Space shift keying modulation for MIMO channels," IEEE Trans. Wireless Commun., vol. 8, no. 7, pp. 3692-3703, July. 2009.

[17] M. Di Renzo, H. Haas, and M. P. Grant, "Spatial modulation for multiple-antenna wireless systems: a survey," IEEE Commun. Mag., vol. 49, pp. 182-191, Dec. 2011.

[18] M. Di Renzo, H. Haas, A. Ghrayeb, S. Sugiura, and L. Hanzo, "Spatial modulation for generalized MIMO: challenges, opportunities, and implementation," Proc. IEEE, vol. 102, no. 1, pp. 56-103, Jan. 2014.

[19] A. Younis, N. Serafimovski, R. Mesleh, and H. Haas, "Generalised spatial modulation," in Proc. ASILOMAR'11, Pacific Grove, USA, Nov. 2010, pp. 1498-1502.

[20] J. T. Wang, S. Y. Jia, and J. Song, "Generalised spatial modulation system with multiple active transmit antennas and low complexity detection scheme," IEEE Trans. Wireless Commun., vol. 11, no. 4, pp. 1605-1615, Apr. 2009.

[21] M. Di Renzo and H. Haas, "On transmit diversity for spatial modulation MIMO: impact of spatial constellation diagram and shaping Filters at the transmitter," IEEE Trans. Veh. Technol., vol. 62, no. 6, pp. 2507-2531, July 2013.

[22] H. Liu. Fu, J. P. Zheng, and Y. Li, "Combining spatial modulation and VBLAST/STBC for correlated Rayleigh fading channels: Capacity and performance analysis," in Proc. WCSP'13, Hangzhou, China, Oct. 2013, pp. $1-5$.

[23] E. Basar, U. Aygolu, E.Panayirci, and H. V. Poor, "Space-time block coded spatial modulation," IEEE Trans. Commun., vol. 59, no. 3, pp. 823 832, Mar. 2011.

[24] R. Mesleh, H. Elgala, and H. Haas, "Optical Spatial Modulation," IEEE/OSA J. Optical Commun. Net., vol. 3, no. 3, pp. 234-244, Mar. 2011.

[25] R. Mesleh, H. Elgala, and H. Haas, "Performance of optical spatial modulation with transmitters-receivers alignment," IEEE Commun. Lett., vol. 15, no. 1, pp. 79-81, Jan. 2011.

[26] C. Briso-Rodriguez, J. M. Cruz, and J. I. Alonso, "Measurements and modeling of distributed antenna systems in railway tunnels," IEEE Trans. Veh. Technol., vol. 56, no. 5, pp. 2870-2879, Sept. 2007.

[27] K. Guan, Z. Zhong, B. Ai, and T. Kürner, "Propagation measurements and analysis for train stations of high-speed railway at $930 \mathrm{MHz}$," IEEE Trans. Veh. Technol., vol. 63, no. 2, pp. 1-16, Feb. 2014.

[28] L. Liu, C, Tao, J, Qiu, H. Chen, L. Yu, W. Dong, and Y. Yuan, "Positionbased modeling for wireless channel on high-speed railway under a viaduct at $2.35 \mathrm{GHz}$," IEEE J. Sel. Areas Commun., vol. 30, no. 4, pp. 834-845, May 2012.

[29] 3GPP, TS36.104, V11.3.1, “3GPP; Technical Specification Group Radio Access Network; E-UTRA; Base Station radio transmission and reception (Release 11),"Jan. 2013.

[30] B. Chen, Z. Zhong, and B. Ai, "Stationarity intervals of time-variant channel in high speed railway scenario," J. China Commun., vol. 9, no. 8, pp. 64-70, Aug. 2012.

[31] R. Parviainen, P. Kyosti, Y. Hsieh, P. Ting, and J. Chiou, "Results of high speed train channel measurements," in COST 2100 TD'08, Lille, France, Oct. 2008

[32] A. Ghazal, C.-X. Wang, H. Haas, M. A. Beach, X. Lu, and D. Yuan, "A non-stationary MIMO channel model for high speed train communication systems," in Proc. IEEE VTC'12-Spring, Yokohama, Japan, May. 2012, pp. $1-5$.

[33] A. Ghazal, C.-X. Wang, H. Haas, M. Beach, R. Mesleh, D. Yuan, X. Ge, and M. K. Chahine, "A non-stationary geometry-based stochastic model for MIMO high-speed train channels," invited paper, in Proc. ITST'12, Taipei, Taiwan, Nov. 2012, pp. 7-11.

[34] A. Ghazal, C.-X. Wang, B. Ai, D. Yuan, and H. Haas, "A nonstationary wideband MIMO channel model for high-mobility intelligent 
transportation systems,” IEEE Trans. Intell. Transp. Syst., vol. 16, no. 2, pp. 885-897, Apr. 2015.

[35] C.-X. Wang, A. Ghazal, B. Ai, P. Fan, and Y. Liu, "Channel measurements and models for high-speed train communication systems: a survey," IEEE Commun. Surveys \& Tutorials, 2016, in press.

[36] M. Di. Renzo and H. Haas, "Bit error probability of SM-MIMO over generalized fading channels," IEEE Trans. Veh. Technol., vol. 61, no. 3, pp. 1124-1144, Mar. 2012.

[37] Y. Fu, C.-X. Wang, Y. Yuan, R. Mesleh, H. Aggoune, M. M. Alwakeel, and H. Haas, "BER performance of spatial modulation systems under 3D V2V MIMO channel models," in IEEE Trans. Veh. Technol., 2016, in press.

[38] E. Basar, U. Aygolu, E. Panayirci, and H. V. Poor, "Performance of spatial modulation in the presence of channel estimation errors," IEEE Commun. Lett., vol. 16, no. 2, pp. 176-179, Feb. 2012.

[39] R. Mesleh and S. S. Ikki, "On the effect of Gaussian imperfect channel estimations on the performance of space modulation techniques," in Proc. IEEE VTC'12-Spring, Yokohama, Japan, May 2012, pp. 1-5.

[40] M. K. Simon and M.-S. Alouini, Digital Communication over Fading Channels, 2nd edition, John Wiley \& Sons, 2005.

[41] M. Di Renzo and H. Haas, "Space shift keying (SSK-) MIMO over correlated Rician fading channels: Performance analysis and a new method for transmit-diversity," IEEE Trans. Commun , vol. 59, no. 1, pp. 116-129, Jan. 2011.

[42] M. Di Renzo and H. Haas, "A general framework for performance analysis of space shift keying (SSK) modulation for MISO correlated Nakagami-m fading channels," IEEE Trans. Commun, vol. 58, no. 9, pp. 2950-2603, Sept. 2010.

[43] A. Hedayat, H. Shah, and A. Nosratinia, "Analysis of space-time coding in correlated fading channels," IEEE Trans. Wireless Commun., vol. 4, no. 6, pp. 2882-2891, Nov. 2005.

[44] A. Forenza, D. J. Love, and R. W. Heath,"A low complexity algorithm to simulate the spatial covariance matrix for clustered MIMO channel models," in Proc. IEEE VTC'04, Miami, USA, May. 2004, pp. 889-893.

[45] S. Sesia, I. Toufik, and M. Baker, LTE - The UMTS Long Term Evolution: From Theory to Practice, John Wiley \& Sons, 2009.

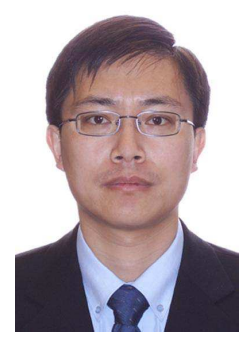

Cheng-Xiang Wang (S'01-M'05-SM'08) received the BSc and MEng degrees in Communication and Information Systems from Shandong University, China, in 1997 and 2000, respectively, and the $\mathrm{PhD}$ degree in Wireless Communications from Aalborg University, Denmark, in 2004.

He has been with Heriot-Watt University, Edinburgh, U.K., since 2005 and was promoted to a Professor in 2011. He is also an Honorary Fellow of the University of Edinburgh, U.K., and a Chair/Guest Professor of Shandong University and Southeast University, China. He was a Research Fellow at the University of Agder, Grimstad, Norway, from 2001-2005, a Visiting Researcher at Siemens AGMobile Phones, Munich, Germany, in 2004, and a Research Assistant at Technical University of Hamburg-Harburg, Hamburg, Germany, from 20002001. His current research interests include wireless channel modeling and 5G wireless communication networks, including green communications, cognitive radio networks, high mobility communication networks, massive MIMO, millimeter wave communications, and visible light communications. He has published 1 book, 1 book chapter, and over 240 papers in refereed journals and conference proceedings.

Prof. Wang served or is currently serving as an editor for 9 international journals, including the IEEE TRANSACTIONS ON VEHICULAR TECHNOLOGY (since 2011), IEEE TRANSACTIONS ON COMMUNICATIONS (since 2015), and IEEE TRANSACTIONS ON WIRELESS COMMUNICATIONS (2007-2009). He was the leading Guest Editor for IEEE Journal on Selected Areas in Communications, Special Issue on Vehicular Communications and Networks. He served or is serving as a TPC member, TPC Chair, and General Chair for over 80 international conferences. He received the Best Paper Awards from IEEE Globecom 2010, IEEE ICCT 2011, ITST 2012, IEEE VTC 2013-Fall, and IWCMC 2015. He is a Fellow of the IET, a Fellow of the HEA, and a member of EPSRC Peer Review College.

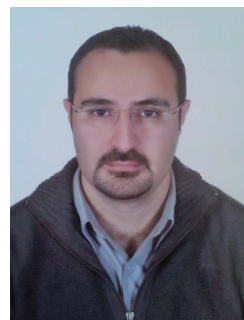

Ammar Ghazal received the BSc degree in Electronics and Telecommunication Engineering from Damascus University, Syria, in 2006 and MSc by Research from Heriot-Watt University, Edinburgh, U.K., in 2010. He received his PhD degree in Electric Engineering from Heriot-Watt University, Edinburgh, U.K., in 2015.

His main research interests include wireless propagation channel characterization and modeling, nonstationary channel models, and high-speed train wireless communications.

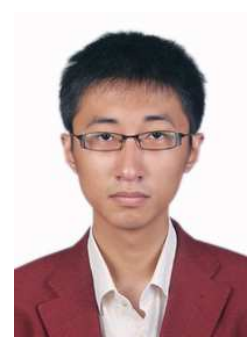

Yu Fu received the BSc degree in Computer Science from Huaqiao University, Fujian, China, in 2009, the MSc degree in Information Technology (Mobile Communications) from Heriot-Watt University, Edinburgh, U.K., in 2010, and the PhD degree in Wireless Communications from Heriot-Watt University, Edinburgh, U.K., in 2015.

$\mathrm{He}$ has been a Postdoc Research Associate of Heriot-Watt University since 2015. His main research interests include advanced MIMO communication technologies, wireless channel modeling and simulation, RF tests, and software defined networks.

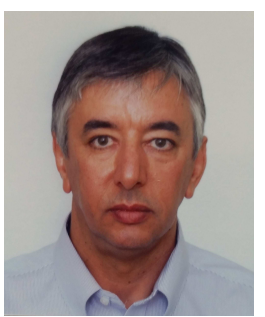

el-Hadi M. Aggoune (M'83-SM'93) received his MS and Ph.D. Degrees in Electrical Engineering from the University of Washington (UW), Seattle, USA. He is a Professional Engineer registered in the State of Washington, and Senior Member of the Institute of the IEEE. He has taught graduate and undergraduate courses in Electrical Engineering at a number of universities in the US and abroad. He served at many academic ranks including Endowed Chair Professor and Vice President and Provost. He was the winner of the Boeing Supplier Excellence Award. He was also the winner of the IEEE Professor of the Year Award, UW Branch. He is listed as Inventor in a major patent assigned to the Boeing Company. His research work is referred to in many patents including patents assigned to ABB, Switzerland and EPRI, USA. Currently he is a Professor and Director of the Sensor Networks and Cellular Systems (SNCS) Research Center, University of Tabuk, Tabuk, Saudi Arabia. He authored many papers in IEEE and other journals and conferences. He is serving on many technical committees. His research interests include modeling and simulation of large scale networks, sensors and sensor networks, scientific visualization, and control and energy systems. 


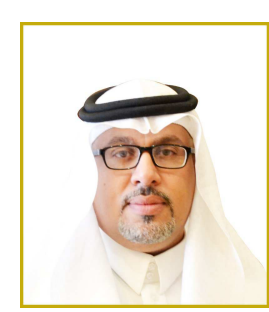

Mohammed M. Alwakeel (SM'14) was born in Tabuk, Saudi Arabia. He received his B.S. and M.S

Degrees from King Saud University, Riyadh, Saudi Arabia, and his Ph.D. Degree in Electrical Engineering from Florida Atlantic University, Boca Raton, Florida. He served as Communications Network Manager at the Saudi National Information Center in Riyadh, and then he served as faculty member at King Abdulaziz University, and then as an Associate Professor and Dean of the Computers and Information Technology College at the University of Tabuk, Tabuk, Saudi Arabia. Currently, he is the Vice Rector for Development and Quality at the University of Tabuk. His current research interests include teletraffic analysis, mobile satellite communications, and sensor networks and cellular systems. 